\title{
双歧杆菌三联活菌胶囊在幽门螺旋杆菌根除治疗中的临 床应用
}

\author{
张 庆 $^{1 *}$ 熊 岗 $^{2}$ 吴新珍 $^{3}$ \\ 1. 孙渡街道卫生院, 江西 331100 \\ 2. 丰城市人民医院, 江西 331100 \\ 3. 丰城市中医院, 江西 331100
}

\begin{abstract}
摘 要: 目的：观察双歧杆菌三联活菌胶囊对幽门螺杆菌（ $\mathrm{Hp}$ ) 的治疗作用。方法：C14呼气试验; 选感染 者 120 例, 随机分为二组; 对照组传统三联治疗, 试验组加双歧杆菌三联活菌胶囊, 测定治疗前、后胃液 $\mathrm{pH}$ 值; 观察不良反应; 复查 $\mathrm{Hp}$ 。结果: 治疗后 $\mathrm{pH}$ 值均较升高 $(P<0.05)$, 有统计学意义; 都有不良反应, 对照组 14 例 (23.33\%)，试验组8例（13.33\%），治疗组明显低于对照组；复查Hp，治疗组根治率为 $81.67 \%$ 显著高于对照组根 除率为 $60 \%,(P<0.05)$ 。结论: 运用传统三联加用双歧杆菌三联活菌胶囊, 能提高Hp的根除率, 减少不良反应并 改善患者胃液 $\mathrm{pH}$ 值。
\end{abstract}

关键词：双歧杆菌三联活菌胶囊；幽门螺杆菌；慢性胃炎；消化性溃病

\section{The Clinical Application of Bifidobacterium Triple Viable Capsules in Eradication of Helicobacter Pylori}

\author{
Qing Zhang ${ }^{1 *}$, Lan Xiong ${ }^{2}$, Xin-Zhen $\mathrm{Wu}^{3}$ \\ 1. Sundu Sub-street Health Center, Fengcheng 331100, Jiangxi, China \\ 2. Fengcheng People's Hospital, Fengcheng 331100, Jiangxi, China \\ 3. Fengcheng Hospital of Traditional Chinese Medicine, Fengcheng 331100, Jiangxi, China
}

\begin{abstract}
Objective: To observe the treatment effect of Bifidobacterium Triple Viable capsules in Helicobacter Pylori(Hp). Method: $\mathrm{C} 14$ breath test. 120 infected patients were randomly divided into two groups. The control group was treated with traditional triple therapy, and the experimental group was treated with Bifidobacterium triple viable capsules. The $\mathrm{pH}$ value of gastric juice before and after treatment was measured; adverse reactions were observed; Hp was reexamined. Result: After treatment, the $\mathrm{PH}$ value both increased $(P<0.05)$ and it has statistical significance; there were adverse reactions in both groups: 14 cases $(23.33 \%)$ in the control group and 8 cases $(13.33 \%)$ in the experimental group. The rate of the experimental group is obviously lower than that of the control group; re-examination of Hp showed that the radical cure rate of the experimental group was $81.67 \%$, which was significantly higher than that of the control group $(60 \%)(P<0.05)$. Conclusion: Traditional triple therapy plus Bifidobacterium triple viable capsules can improve HP eradication rate, reduce adverse reactions and improve gastric juice $\mathrm{PH}$ value.
\end{abstract}

Keywords: Bifidobacterium Triple Viable capsules; Helicobacter Pylori; chronic gastritis; pepticulcer

\section{一, 前言}

研究表明, 幽门螺旋杆菌 ( Hp ) 感染与慢性胃炎、十二指肠溃疡、胃癌、胃溃疡及胃黏膜淋巴组织相关淋巴瘤的 发生密切相关, 1996年WHO癌症研究机构把幽门螺旋杆菌 ( Hp ) 化为 I类生物致癌因子 ${ }^{[1]}$ 。当前幽门螺旋杆菌 $(\mathrm{Hp})$

*通讯作者: 张庆, 1982年10月, 男, 汉族, 江西丰城人, 现任职于孙渡街道卫生院消化内科, 副主任医师, 本 科，研究方向：消化系常见疾病，胃肠病。 
在全世界发展中国家人群中的感染率比较高，部分发展中国家的成人幽门螺旋杆菌（Hp）的感染率甚至高达 $90 \%{ }^{[2]}$ 。 根除幽门螺旋杆菌 ( Hp ) Hp感染的治疗, 不仅能治疗和预防消化系统疾病, 也有益于其他系统相关疾病的预防与治 疗。既往根除幽门螺旋杆菌 ( Hp ) 的治疗方案主要是质子原抑制剂联合抗生素, 随着幽门螺旋杆菌 ( Hp ) 耐药菌株 的出现, 幽门螺旋杆菌 ( $\mathrm{Hp}$ ) 的根治越来越困难。益生菌具有补充肠道益生菌, 提高机体免疫力、促进肠秥膜修复、 增强肠黏膜屏障及防治多种疾病的作用, 近年来作为主要的辅助药物应用于幽门螺旋杆菌 ( Hp ) 治疗 ${ }^{[3]}$ 。我们自 2014 年11月至2017年12月共检测出800余例幽门螺杆菌 ( HP) 阳性患者, 对其中 120 例反复上腹部不适及幽门螺杆菌持续阳 性患者在联合其他药物基础上，选择恰当剂量双歧杆菌三联活菌，能有效降低不良反应的发生率，提高治疗幽门螺旋 杆菌（Hp）根除率; 改善胃肠道功能，现介绍如下。

\section{二、资料与方法}

(一) 一般资料

选取2014年11月至2017年12月丰城市孙渡街道卫生院检测的Hp感染患者120例, 在120例慢性胃炎及胃十二指肠溃 疡感染幽门螺旋杆菌患者中, 男性55例, 女性65例, 年龄18至85岁, 平均 $(58.2 \pm 7.5)$ 岁。患者反复上腹胀痛, 饱胀 感，进食后饱胀感加重，中上腹不同程度压痛，在我院的胃镜检查：慢性胃炎伴様烂的 70例（占58.33\%）; 胃溃疡23 例（占 $19.17 \%$ ); 十二指肠溃疡 22 例（占 $18.33 \%$ )，其他 5 例 $(4.17 \%$ )，按人院时间先后将患者随机分为对照组和 观察组各60例。

\section{(二) 方法}

对照组采用三联疗法：阿莫西林胶囊（珠海联邦制药股份有限公司中山分公司，0.5克/粒） 2 粒, 每天两次, 每 次两粒; 泮托拉唑钠肠溶胶囊（湖南华纳大药厂股份有限公司，40毫克/片），每天一次，每次1片; 克拉䨖素分散片 （扬子江药业集团有限公司，0.25克/片），每天 2 次，每次 1 片。治疗 14 天为 1 疗程。

实验组同样采用三联疗法, 并加用双歧杆菌三联活菌胶囊 (上海信谊药厂有限公司, 0.21 克/粒), 每天三次, 每 次三粒，与抗生素服药时间间隔 $2 \mathrm{~h}$ 以上，

(三) 观察指标

由于两组患者采用不同的治疗方案, 因此需要通过治疗前后的胃液 $\mathrm{pH}$ 值比对以及患者治疗后的不良反应率的比对 探究不同治疗方案的临床价值, 并最终观察患者4周以后的幽门螺杆菌根除率。

(四) 统计学处理

研究获取患者资料采用SPSS 18.0 系统软件分析; 计量资料用 $(x \pm s)$ 表示, 并用 $t$ 检验; 计数资料用 $(n, \%)$ 表 示, 并用 $x^{2}$ 检验; $P<0.05$ 表示有统计学意义。

\section{三、结果}

(一) 两组治疗前后胃液 $\mathrm{pH}$ 值对比

经过相关药物治疗后， 2 组患者治疗后四周胃液的 $\mathrm{pH}$ 值均有明显升高，差异有统计学意义， $P<0.05$ 。见表 1 。

表1 两组患者的两组治疗前后胃液 $\mathrm{pH}$ 值

\begin{tabular}{ccccc}
\hline 组别 & 例数 & 治疗前 & 治疗后4周 & $P$ \\
\hline 对照组 & 60 & $1.51 \pm 0.72$ & $4.22 \pm 0.57$ & $<0.05$ \\
实验组 & 60 & $1.49 \pm 0.59$ & $4.24 \pm 0.68$ & $<0.05$ \\
$P$ & - & $>0.05$ & $>0.05$ & \\
\hline
\end{tabular}

(二) 两组不良反应比较

在治疗过程中，两组患者都有不同程度的不良反应现象，对照组14例（23.33\%），试验组8例（13.33\%）, 两组 有统计学意义 $(P<0.05)$ 。见表2。

表2 两组患者的不良反应

\begin{tabular}{ccc}
\hline 组别 & 例数 & 不良反应 $(\%)$ \\
\hline 实验组 & 60 & $8(13.33 . \%)$ \\
对照组 & 60 & $14(23.33 \%)$ \\
$P$ & - & $P<0.05$ \\
\hline
\end{tabular}


(三) $\mathrm{Hp}$ 根除率比较

治疗后 4 周复查 $14 \mathrm{C}$ 尿素呼气试验, 对照组的幽门螺杆菌根除率为 $60 \%$ 明显优于对照组根除率为 $81.67 \%$, 差异 有统计学意义 $(\mathrm{P}<0.05)$ 。见表3。

表3 两组患者的 $\mathrm{Hp}$ 根除率比较

\begin{tabular}{ccc}
\hline 组别 & 例数 & HP根除率 $(\%)$ \\
\hline 实验组 & 60 & $49(81.67 \%)$ \\
对照组 & 60 & $36(60 \%)$ \\
$P$ & - & $P<0.05$ \\
\hline
\end{tabular}

\section{四、讨论}

H.pylori为革兰阴性杆菌, 微需氧, 电镜下, 未端钝圆、螺旋形弯曲的细菌, 菌体一端伸出 $2 \sim 6$ 条带鞘的鞭毛。 长2.5 4.0 $\mu \mathrm{m}$ ，宽0.5 $1.0 \mu \mathrm{m}$ 。鞭毛在运动中起推进器作用，在定居过程中起抛针作用，寄居在人体胃部表面。

人类是H.pylori是唯一自然宿主，全世界自然人群H.pylori感染率高达 $50 \%$ ，中国自然人群H.pylori感染率约 $54.7 \%$ ，血清阳性范围 40-90\%（平均59\%）；在我国调查显示幽门螺旋杆菌（ Hp）感染率为 $60 \% \sim 70 \%{ }^{[4]}$ 。 Hp感染与 消化性溃疡、胃癌、慢性胃炎、胃黏膜相关淋巴样组织淋巴瘤等消化系统疾相关。随着Hp对抗生素耐药菌株的出现, 质子泵抑制剂联合抗生素标准三联或四联方案的疗效逐渐下降, 为了提高幽门螺旋杆菌的根除率, 延长服药时间, 但 是长期服用抗生素容易使肠道菌群失衡, 很可能破坏肠道微生态屏障, 还可能引发腹泻、腹痛、便秘、恶心、呕吐等 不良反应，有些患者由于对不良反应不能耐受，不能坚持服药，甚至自行停药，从而导致治疗的失败。

幽门螺杆菌是一种极易在人与人之间传播的革兰氏阴性螺旋状细菌, 从口腔进人人体后可定植于胃肠型上皮, 难 以自发清除, 很容易造成持久性或终生感染, 且感染后可导致消化不良、慢性胃肠炎及溃疡、胃癌、胃粘膜相关淋巴 组织淋巴瘤以及胃外疾病等; 该疾病严重影响人们的日常工作和生活。《幽门螺杆菌感染基层诊疗指南》指出, 两大 类人群强烈推荐根除Hp，另有十大类人群推荐根除，临床工作中可根据表4查找判断：

表4 幽门螺杆菌根除指征

\begin{tabular}{|c|c|c|}
\hline 幽门螺杆菌的阳性疾病 & 强烈推荐 & 推荐 \\
\hline 消化性溃疡 (不论是否活动和有无并发症） & V & \\
\hline 胃粘膜相关淋巴组织 (MALT) 淋巴瘤 & $\sqrt{ }$ & \\
\hline 慢性胃炎伴消化不良症状 & & V \\
\hline 慢性胃炎伴胃粘膜萎缩或糜炬 & & $\checkmark$ \\
\hline 早期胃肿瘤已行内镜下切除或手术胃次全切除 & & 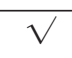 \\
\hline 长期服用质子泵抑制剂 & & $\checkmark$ \\
\hline 胃癌家族史 & & $\checkmark$ \\
\hline 计划长期服用NSAID（包括低剂量阿司匹林） & & $\checkmark$ \\
\hline 不明原因缺铁性贫血 & & $\checkmark$ \\
\hline 特发性血小板减少性紫癜（ITP） & & $\checkmark$ \\
\hline $\begin{array}{c}\text { 其他幽门螺杆菌相关性疾病 (如淋巴细胞增生性 } \\
\text { 胃炎、增生性胃息肉 ) }\end{array}$ & & V \\
\hline 个人要求治疗 & & $\checkmark$ \\
\hline
\end{tabular}

因此，根除幽门螺杆菌（Hp），从而提高治疗有效率，在缓解症状、减轻疼痛、节省费用等方面具有重要意义; 那么, 怎样根除幽门螺旋杆菌 ( Hp ) 感染一直是近年来医疗界研究的难点和热点, 加益生菌制剂的疗法受到了广泛关 注, 益生菌具有补充肠道益生菌的作用, 并且能够提高机体免疫力、促进肠黏膜修复、增强肠黏膜屏障, 还可以预防 和治疗多种疾病, 近年来作为主要的辅助药物应用于幽门螺旋杆菌 ( $\mathrm{Hp}$ ) 治疗, 其中最为广泛就是双歧杆菌三联活菌 胶囊的应用, 并取得了良好好的临床疗效 ${ }^{[5]}$ 。双歧杆菌三联活菌胶囊 ${ }^{[6]}$ 中包含多种有益菌, 其中最主要的嗜酸性乳杆 菌繁殖较快, 同样人体肠道主要的益生菌, 由于本身具有高耐酸性, 还对多种抗生素具有耐性, 自身繁殖产生的乳酸 能够抑制尿素酶活性, 从而抑制和杀灭幽门螺旋杆菌 ( Hp ) ; 肠球菌的活性不受胃肠道酸性环境的影响, 而且能够降 低尿素酶活性, 从而抑制幽门螺旋杆菌 ( $\mathrm{Hp}$ ) 的生长, 保护胃黏膜; 双歧杆菌能够抑制幽门螺旋杆菌 ( $\mathrm{Hp})$ 生长和 
繁殖, 对而药的幽门螺旋杆菌 ( Hp ) 具有明显的抑制作用, 且可以分泌热稳定活性蛋白。三联疗法联合双歧杆菌三联 活菌胶囊可增强幽门螺旋杆菌 ( $\mathrm{Hp}$ ) 的根除率, 减少不良反应, 提高患者的医从性, 从而缓解患者的症状, 改善胃肠 道功能、缩短服药时间等方面取得满意的效果。

本研究采用三联疗法联合双歧杆菌三联活菌胶囊在幽门螺旋杆菌根除治疗中发现, 对照组的幽门螺杆菌根除率为 $60 \%$, 治疗组根除率为 $81.67 \%$, 两组在治疗过程中均出现不同程度的不良反应, 对照组 14 例 $(23.33 \%$ ）, 试验组 8 例 $(13.33 \%)$, 两组差异有统计学意义。从本研究可以看出采用双歧杆菌三联活菌胶囊得实验组不仅提高了幽门螺杆菌 的根除率, 而且减少了不良反应, 这样可使患者的医从性增加, 这与近几年联合双歧杆菌根除幽门螺杆菌治疗的文献 结果相似 ${ }^{[7]}$ 。提示三联疗法联合双歧杆菌三联活菌胶囊治疗幽门螺杆菌感染的疗效明显好于单用三联疗法的治疗。

双歧杆菌三联活菌胶囊在幽门螺杆菌治疗中的临床应用: 益生菌提高幽门螺杆菌根除效率的可能机制：第一， 免疫原性引发的主动免疫机制; 第二, 胃肠道功能改善, 有利于Hp清除; 第三, 益生菌在胃内定植, 提高 Hp的根除 率; 第四, 缓解Hp对胃黏膜损伤, 减少炎症相关因子; 益生菌用于辅助根除幽门螺杆菌的收益: 第一, 提高根除率 8\% 13\%（无论三联还是四联方）; 第二，胃肠道功能改善减少根除方案相关的胃肠道反应 $13.8 \% （ 10 \%$ 15\% ）; 第三，降低根除方案所致的肠道微生态紊乱；第四，单用益生菌制剂致幽门螺杆菌检测阴性的效率约 $60 \%$ 。

\section{五、结论}

综上所述, 采用三联疗法联合双歧杆菌三联活菌胶囊根除幽门螺旋杆菌的治疗方案具有疗效强、安全性高, 不良 反应少的特点, 患者耐受性好, 医从性好强, 更加适合于临床。但本研究由于条件所限, 有其局限性, 未能随访患者 的复发率, 且样本比较少、仍需进一步行大样本、多中心的验证, 以便临床广泛推广, 为彻底根除幽门螺杆菌的最佳 治疗方案提供合理的依据。

\section{参考文献:}

[1]Jagusztyn-Krynicka EK,Godlewska R.New approaches for Helicobacter vaccine development-difficulties and progress[J].Pol J Microbiol, 2008,57(1):3-9.

[2] Franceschi F,Gasbarrini A.Helicobacter pylori and extra-gas-tric diseasdes[J].Best Pract Res Clin Gastroenterol, 2007.21(2):325-334.

[3] 阎澜,张军东.培菲康临床应用进展[J].上海医药, 2007,28(3):114-116.

[4]李竟,袁媛,黄或等.健康人群幽门螺杆菌感染率调查分析[J].蚌埠医学院学报, 2012,37(11):1341-1344.

[5]刘留,费素娟. 双歧杆菌三联活菌胶囊辅助根除幽门螺杆菌感染临床疗效的荟萃分析 [J].药学实践杂志, 2016,34(4): 380-384.

[6]张莉.双歧杆菌活菌胶囊联合质子永抑制剂三联疗法治疗幽门螺杆菌阳性消化性溃疡 90 例 [J].中国药业, 2014,23 (1):75-77.

[7]付尚勤,谢步善,万伦常.益生菌联合标准三联疗法治疗 Hp 阳性消化性溃疡的疗效[J]. 实用临床医药杂志, 2014,18 (13):111-112. 